\title{
A UTILIZAÇÃO DE PLANTAS MEDICINAIS POR PACIENTES COM DOENÇAS RESPIRATÓRIAS CRÔNICAS PARTICIPANTES DO GRUPO DE REABILITAÇÃO PULMONAR
}

The use of medicinal plants in patients with chronic respiratory diseases participants of a pulmonary rehabilitation group

Daniela da Silva Lumertz¹, Angela Erna Rossato², Ioná Vieira Bez Birollo³, Maria Tereza Soratto 4

${ }^{1}$ Enfermeira. Universidade do Extremo Sul Catarinense, Criciúma/SC, Brasil.

${ }^{2}$ Mestrado em Farmácia. Professora do Curso de Farmácia, Universidade do Extremo Sul Catarinense, Criciúma/SC, Brasil.

${ }^{3}$ Mestre em Enfermagem. Professora do Curso de Enfermagem, Universidade do Extremo Sul Catarinense, Criciúma/SC, Brasil.

${ }^{4}$ Mestre em Educação. Professora do Curso de Enfermagem, Universidade do Extremo Sul Catarinense, Criciúma/SC, Brasil.

\section{Endereço para correspondência:}

Maria Tereza Soratto

Av. Universitária, 1105 - Curso de Enfermagem. Bloco S. Bairro Universitário, Criciúma/SC.

CEP: 88806-000

Email: guiga@unesc.net 


\title{
Resumo
}

Estudo com objetivo de identificar as plantas medicinais utilizadas por pacientes com doenças respiratórias crônicas participantes de um grupo de reabilitação pulmonar. Pesquisa de abordagem qualitativa, descritiva, exploratória e de campo. Aplicou-se entrevista semiestruturada com 10 pacientes do Grupo de Reabilitação Pulmonar da Clínica de Fisioterapia da Universidade Extremo Sul Catarinense - UNESC. A análise dos dados foi realizada com a análise de conteúdo. As principais plantas utilizadas pelos pacientes que participam do Grupo de Reabilitação Pulmonar foram camomila, boldo, guaco, cidreira, hortelã e folha de laranja. O conhecimento sobre a utilização das plantas medicinais advém principalmente da cultura passada de geração em geração pelas famílias. Os pacientes em sua maioria não receberam orientação da equipe multiprofissional sobre a utilização das plantas medicinais e interação medicamentosa. A forma utilizada das Plantas medicinais pelos pacientes geralmente está de acordo com as indicações da Agência Nacional de Vigilância Sanitária. Os pacientes demonstraram dificuldade para relatar como ocorre a preparação das plantas medicinais. Considera-se primordial a revalorização do uso de plantas medicinais pela equipe multiprofissional de saúde, em concordância com as diretrizes da Política Nacional de Práticas Integrativas e Complementares no Sistema Único de Saúde e Programa Nacional de Plantas Medicinal e Fitoterápico. Sugere-se pesquisas direcionadas à atuação dos profissionais da saúde voltados às Plantas Medicinais e Fitoterápicas.

Palavras-chave: Plantas Medicinais; Doença Crônica; Doenças Respiratórias; Reabilitação.

\begin{abstract}
Study in order to identify medicinal plants used for patients with chronic respiratory diseases participants of a pulmonary rehabilitation group. Research of qualitative, descriptive, exploratory approach and field. Applied semi-structured interview with 10 patients of pulmonary rehabilitation clinic group of physiotherapy at the University of Southern Santa Catarina. Data analysis was performed using content analysis. The main plants used by patients participating in the Group of Pulmonary Rehabilitation have been chamomile, boldo, guaco, lemon balm, mint and orange sheet. The
\end{abstract}


Artigo Original

Atenção à Saúde

knowledge about the use of medicinal plants comes mainly from the culture passed on from generation to generation by families. Your most patients did not receive guidance from multidisciplinary team on the use of medicinal plants and drug interaction. The form of medicinal plants used by patients is usually according to the indications of the National Health Surveillance Agency. The patients demonstrated difficulty to report as the preparation of medicinal plants. It is considered crucial to upgrading the use of medicinal plants by the multidisciplinary team of health in accordance with the guidelines of the national policy of Integrative and complementary practices in the unique system of health and National Program of Medicinal Plants and herbal medicines. Suggested research directed the activities of health professionals focused on medicinal plants and herbal remedies.

Keywords: Plants; Medicinal; Chronic Disease; Respiratory Tract Diseases; Rehabilitation.

\section{INTRODUÇÃO}

Doenças respiratórias crônicas (DRC) são doenças crônicas tanto das vias aéreas superiores como das inferiores. A asma, a rinite alérgica e a doença pulmonar obstrutiva crônica (DPOC) são as DRC mais comuns. Representam um dos maiores problemas de saúde mundialmente ${ }^{1}$.

O Programa Nacional de Plantas Medicinais e Fitoterápicos - PNPMF tem como princípios a ampliação das opções terapêuticas e a melhoria da atenção à saúde aos usuários do SUS mediante a inserção das plantas medicinais e medicamentos fitoterápicos nos serviços do sistema ${ }^{2}$. A fitoterapia caracteriza-se pela utilização de plantas medicinais com formas farmacêuticas diferenciadas, sem a utilização de substâncias ativas isoladas ${ }^{3}$.

O Formulário de Fitoterápicos da Farmacopéia Brasileira ${ }^{4}$ organizou uma lista de plantas medicinais com as indicações e contra-indicações, modo de uso para a utilização na rede de assistência e pelos profissionais de saúde. Dentre as plantas medicinais indicadas para doenças respiratórias encontra-se Guaco, Eucalipto, Alcaçuz, Malva, Poejo, Polígala e Assapeixe. 
Conforme a Política Nacional de Práticas Interativas e Complementares no SUS ${ }^{5}$, devem ser tomadas medidas que possibilitem tornar disponíveis plantas medicinais e fitoterápicas nas unidades de saúde, utilizando um ou mais produtos, como: planta medicinal in natura, planta medicinal seca, fitoterápico manipulado e fitoterápico industrializado. As espécies utilizadas devem ser fornecidas, utilizando as espécies identificadas botanicamente, tendo garantia das boas práticas de cultivo, implantação e manutenção de hortos oficiais, oferecendo também local adequado para 0 armazenamento das drogas vegetais 5 .

O Brasil é um país com diversificada biodiversidade, que, associada a uma rica diversidade étnica e cultural, detém um valioso conhecimento tradicional associado ao uso de plantas medicinais ${ }^{6}$, tendo 0 potencial necessário para desenvolvimento de pesquisas com resultados em tecnologias e terapêuticas apropriadas $^{6,7}$.

O profissional enfermeiro deve considerar a prática da utilização de plantas medicinais como sendo um cuidado singular e integral centrado na cultura e nas crenças da população ${ }^{8}$. Nesta perspectiva, pretende-se com este estudo conhecer de que forma os pacientes com doenças respiratórias crônicas utilizam as plantas medicinais, e se a utilização está de acordo com as indicações do Formulário de Fitoterápicos da Farmacopéia Brasileira ${ }^{4,9}$.

A Política de Práticas Integrativas e Complementares e o Programa Nacional de Plantas Medicinais e Fitoterápicos recomendam a utilização dos fitoterápicos na rede do SUS, incentivando a capacitação permanente da equipe de saúde nesta área. Considera-se extremamente necessário uma rede de cuidados integrais relacionados às doenças crônicas, para buscar uma melhor qualidade de vida do paciente, e diminuir os riscos de complicações ${ }^{2,3}$.

A presente pesquisa tem como objetivo verificar se as plantas medicinais utilizadas por pacientes com doenças respiratórias crônicas participantes de um grupo de reabilitação pulmonar estão de acordo com as indicações da Farmacopéia Brasileira.

\section{MÉTODOS}


Pesquisa de abordagem qualitativa, descritiva, exploratória e de campo. $\mathrm{O}$ estudo foi desenvolvido na Universidade do Extremo Sul Catarinense. Aplicou-se entrevista semiestruturada com 10 pacientes do Grupo de Reabilitação Pulmonar da Clínica de Fisioterapia da UNESC. Utilizaram-se como critérios de inclusão: pacientes que participam do Grupo de Reabilitação Pulmonar e que utilizam plantas medicinais; aceitação para participar da Pesquisa, segundo Resolução 466/12 ${ }^{10}$.

A análise dos dados foi realizada a partir da análise de conteúdo com a categorização dos dados, através da ordenação, classificação e análise final dos dados pesquisados ${ }^{11}$.

Para preservar o sigilo decorrente das entrevistas realizadas, de acordo com as diretrizes e normas regulamentadoras da Resolução 466/12 ${ }^{10}$, que envolvem pesquisa com Seres Humanos e Grupos Vulneráveis, utilizou-se a letra "P" para os pacientes, seguido do respectivo número - $\mathrm{P} 1$ a $\mathrm{P} 10$. A pesquisa foi aprovada pelo Comitê de Ética em Pesquisa da UNESC, pelo no Projeto 꾸 1.342.991/2016.

\section{RESULTADOS E DISCUSSÃO}

Em relação ao perfil dos pacientes entrevistados, cinco são do sexo masculino e cinco são do sexo feminino, a idade variou de 59 anos a 79 anos, cinco são casados, três são viúvas, uma solteira e uma divorciada, sendo que todos os pacientes participaram integralmente da pesquisa.

Quadro 1. Caracterização do perfil dos pacientes.

\begin{tabular}{|c|c|c|c|c|}
\hline Paciente & Sexo & Idade & Tipo de doença respiratória & $\begin{array}{c}\text { Tempo de } \\
\text { adoecimento }\end{array}$ \\
\hline P1 & M & $\begin{array}{c}76 \\
\text { anos }\end{array}$ & Pneumoconiose, pneumonia & 20 anos \\
\hline P2 & F & $\begin{array}{c}62 \\
\text { anos }\end{array}$ & $\begin{array}{c}\text { Doença Pulmonar Obstrutiva Crônica } \\
\text { (DPOC) }\end{array}$ & 10 anos \\
\hline P3 & F & $\begin{array}{c}79 \\
\text { anos }\end{array}$ & $\begin{array}{r}\text { Doença Pulmonar Obstrutiva Crônica } \\
\text { (DPOC) e Enfisema Pulmonar }\end{array}$ & 40 anos \\
\hline P4 & M & 59 & Doença Pulmonar Obstrutiva Crônica & 10 anos \\
\hline
\end{tabular}


Artigo Original

\section{Atenção à Saúde}

\begin{tabular}{|c|c|c|c|c|}
\hline Paciente & Sexo & Idade & Tipo de doença respiratória & $\begin{array}{c}\text { Tempo de } \\
\text { adoecimento }\end{array}$ \\
\hline P5 & F & $\begin{array}{c}67 \\
\text { anos }\end{array}$ & (DPOC) & 10 anos \\
\hline P6 & M & $\begin{array}{c}77 \\
\text { anos }\end{array}$ & Enfisema Pulmonar & 16 anos \\
\hline P7 & F & $\begin{array}{c}59 \\
\text { anos }\end{array}$ & Enfisema Pulmonar & 5 anos \\
\hline P8 & M & $\begin{array}{c}74 \\
\text { anos }\end{array}$ & $\begin{array}{r}\text { Doença Pulmonar Obstrutiva Crônica } \\
\text { (DPOC) }\end{array}$ & 20 anos \\
\hline P9 & F & $\begin{array}{c}72 \\
\text { anos }\end{array}$ & $\begin{array}{r}\text { Doença Pulmonar Obstrutiva Crônica } \\
\text { (DPOC) e Asma }\end{array}$ & 2 anos \\
\hline P10 & $\mathrm{M}$ & $\begin{array}{r}66 \\
\text { anos }\end{array}$ & $\begin{array}{r}\text { Doença Pulmonar Obstrutiva Crônica } \\
\text { (DPOC) }\end{array}$ & \\
\hline
\end{tabular}

Fonte: Dados de pesquisa, 2016.

O tempo de adoecimento dos pacientes variou de 2 a 40 anos, tempo característico de doença crônica, que pode ser definida por uma doença com início gradual, de prognóstico usualmente incerto, com longa ou indefinida duração ${ }^{12}$.

Usualmente as doenças crônicas apresentam um curso clínico que pode sofrer mudanças com o percurso da doença, com períodos de agudização, podendo gerar incapacidade ao paciente. Essas requerem a utilização de diferentes tipos de tecnologias, que associadas a significativas mudanças no estilo de vida e um processo de cuidado contínuo podem oferecer tratamento aos sintomas, mas nem sempre a cura. As doenças crônicas no Brasil são as responsáveis por grande número das internações, causando problemas relacionados à perda da mobilidade, amputações e prejuízos neurológicos. Além disso, ainda causam uma perda significativa na qualidade de vida, que piora na medida em que a doença vai avançando ${ }^{13}$.

\section{Tipo e tempo de doença respiratória do paciente}


Em relação ao tipo de doença respiratória, os pacientes relataram possuir: DPOC(P2; P3; P4; P8; P9; P10); Enfisema (P3; P5; P6; P7); Asma (P9) e Pneumoconiose associado a Pneumonia $(\mathrm{P} 1)$. Os relatos referentes às doenças respiratórias dos pacientes demonstram em alguns casos a associação de uma ou mais doenças:

\section{P1 - "Tenho Pneumoconiose e Pneumonia". \\ P3 - "Tenho DPOC e Enfisema". \\ P9- "Eu tenho a doença DPOC e Asma".}

As doenças respiratórias crônicas são doenças crônicas tanto das vias aéreas superiores como das inferiores. Representando um grave problema de saúde atualmente, a maioria delas apresenta-se como doenças preveníveis, que podem atingir ambos os sexos e todas as idades. Porém as populações mais atingidas são os extremos de idade, acometendo principalmente crianças e idosos. Alguns fatores de risco já foram reconhecidos e medidas eficazes para a prevenção foram estabelecidas. Entre os fatores de risco estão tabagismo, poluição ambiental, alérgenos, agentes ocupacionais e algumas doenças como esquistossomose e doença falciforme. Doenças que prejudicam o funcionamento, ou causam algum dano às vias aéreas deixando cicatrizes, e o aumento da expectativa de vida também podem ser consideradas como fatores de risco ${ }^{1}$.

O prognóstico, a atenção às manifestações extrapulmonares e comorbidades, escolha da medicação e a consideração a respeito de transplante pulmonar, todos dependem de um diagnóstico e de um estadiamento preciso, já que algumas doenças pulmonares podem apresentar sintomatologias parecidas. Pacientes com doenças pulmonares de diagnóstico incerto devem ser encaminhados para centros de referência, para que seja realizada consulta com especialistas clínicos, radiologistas experientes e patologistas especializados. É frequente a identificação de algumas doenças em associação. Como exemplo, temos o enfisema pulmonar, que pode estar associado à outra patologia do sistema respiratório ${ }^{14}$.

Algumas das doenças respiratórias possuem uma sintomatologia semelhante, evoluindo conforme a gravidade da doença. Na rinite alérgica ocorre 
Artigo Original

Atenção à Saúde

uma resposta decorrente de uma reação inflamatória mediada por anticorpos lgE, manifestada após a exposição a patógenos alérgenos. A rinite alérgica possui uma estreita relação com a asma, interfere no controle da doença e torna o seu manejo sintomático mais difícil, possuindo sintomas clínicos reversíveis espontaneamente ou com tratamento ${ }^{15,16}$.

A doença pulmonar obstrutiva crônica (DPOC), caracterizada pela obstrução crônica e parcialmente reversível do fluxo aéreo, é normalmente progressiva, porém prevenível e tratável. Esta se dá por uma resposta inflamatória anormal dos pulmões devido à inalação de partículas ou gases tóxicos e já pode ser considerada a maior causa crônica de morbimortalidade no mundo ${ }^{17,18}$.

O enfisema pulmonar é conhecido como uma das formas clínicas da DPOC, se caracterizando pelo desenvolvimento progressivo do aprisionamento aéreo, alterações alveolares e hiperinsulflação pulmonar. A evolução da doença é geralmente uma doença pulmonar terminal com necessidade de transplante pulmonar. Quando associado à bronquite crônica, ocasiona a obstrução ao fluxo de ar, evoluindo dessa maneira para doença pulmonar obstrutiva crônica ${ }^{19,20}$.

Já na pneumoconiose, a inalação e o acúmulo de poeira nos pulmões acarretam uma reação pulmonar que pode evoluir, em alguns casos, para fibrose pulmonar difusa. Pode levar anos para se manifestar clinicamente, porém, com a progressão das lesões, há uma redução da complacência pulmonar e limitação das trocas gasosas, fazendo com que o trabalhador queixe-se de dispnéia aos esforços e astenia ${ }^{21}$.

Além das doenças respiratórias, os pacientes relataram possuir principalmente problemas cardíacos (P2; P4; P5; P8; P10); hipertensão (P2; P3; P7; P8); diabetes (P2; P8; P10); problema renal e ansiedade (P5); conforme destacado nas falas:

P2- "Tenho problema no coração, pressão alta e diabetes".

P3 - "Tenho hipertensão".

P5- "Eu tenho problema renal, cardíaco e problema de ansiedade".

P7- "Tenho hipertensão".

P8- "Tenho diabetes, problema cardíaco e hipertensão".

P10- "Tenho problema cardíaco e diabetes". 
De acordo com a Sociedade Brasileira de Cardiologia, a hipertensão arterial sistêmica (HAS) é uma condição clínica multifatorial, caracterizada por níveis elevados e sustentados de pressão arterial. Uma doença crônica de difícil controle e altas taxas de prevalência, que acompanha diversos riscos à saúde ${ }^{22}$.

$\mathrm{Na}$ diabetes mellitus ocorre um transtorno em nível de metabolismo, que é caracterizado por hiperglicemia e distúrbios no metabolismo de carboidratos, proteínas e gorduras, resultantes de defeitos da secreção e/ou da ação da insulina. Com o passar dos anos vem aumentando cada vez mais sua prevalência e está habitualmente associada à outra patologia. Quando tratada de forma efetiva na atenção primaria à saúde, evita-se hospitalizações e complicações que podem levar o paciente ao óbito ${ }^{12}$.

Destaca-se no relato do Paciente P5, além de problema cardíaco, associação de doença renal e ansiedade com a doença respiratória crônica: P5- "Eu tenho problema renal, cardíaco e problema de ansiedade".

A doença renal crônica é uma doença sistêmica que pode causar perda da autonomia do paciente, levando a restrições físicas, limitações e, em alguns casos, perdas sociais. Os tratamentos rigorosos e debilitantes podem causar sérias mudanças no estado psicológico do paciente, trazendo pioras para o estado de saúde. Indivíduos submetidos a tratamentos rigorosos estão mais vulneráveis ao estresse e apresentam maior tendência de reagir a situações ameaçadoras com intensidade mais elevada de ansiedade, pois vivenciam condições particulares diariamente ${ }^{23}$.

Os pacientes $\mathrm{P} 1, \mathrm{P} 6$ e P9 relataram não possuírem doenças associadas, além da doença respiratória crônica.

As doenças respiratórias crônicas são as responsáveis por grandes mudanças na vida dos pacientes, interferindo de maneira negativa na sua qualidade de vida, o deixando suscetível a outras patologias. Quando as comorbidades entram em associação com a doença respiratória crônica ocorre uma mudança brusca no estado de saúde do paciente, o que pode causar uma instabilidade física, emocional e mental ${ }^{1}$.

\section{As plantas medicinais utilizadas pelo paciente para doenças respiratórias}


Artigo Original

Atenção à Saúde

As plantas medicinais utilizadas pelo paciente para doenças respiratórias foram guaco e camomila (04), hortelã (03), folha de laranja (02), além de alho, maracujá e cidreira (01), conforme descrito no Quadro 2.

Quadro 2. Plantas medicinais utilizadas pelos pacientes e indicação segundo Formulário de Farmacopéia Brasileira.

\begin{tabular}{|c|c|c|}
\hline Plantas Medicinais & Pacientes (P) & $\begin{array}{c}\text { Indicação das Plantas conforme } \\
\text { literatura }\end{array}$ \\
\hline $\begin{array}{c}\text { Guaco } \\
\text { Mikania glomerata } \\
\text { Sprengel }\end{array}$ & $\begin{array}{c}04 \text { (P1; P3; P5; } \\
\text { P6) }\end{array}$ & $\begin{array}{l}\text { Expectorante }{ }^{4,9} \text {. } \\
\text { Broncodilatador } 9\end{array}$ \\
\hline $\begin{array}{c}\text { Camomila } \\
\text { MatricariarecutitaL. }\end{array}$ & $\begin{array}{c}04 \text { (P4; P6; P9; } \\
\text { P10) }\end{array}$ & $\begin{array}{l}\text { Antiespasmódico }{ }^{4,9} \text {. Dispepsias } \\
\text { funcionais }{ }^{9} \text {. } \\
\text { Ansiolítico e sedativo leve }{ }^{4} \text {. }\end{array}$ \\
\hline $\begin{array}{c}\text { Hortelã } \\
\text { Menthacitrata Ehrh. }\end{array}$ & 03 (P1; P3; P6) & Antiespasmódico e antiflatulento ${ }^{4}$. \\
\hline $\begin{array}{c}\text { Laranja } \\
\text { Citrusaurantium L. }\end{array}$ & 02 (P1; P2) & Ansiolítico e sedativo leve ${ }^{4}$. \\
\hline $\begin{array}{c}\text { Alho } \\
\text { Alliumsativum L. }\end{array}$ & $01(\mathrm{P} 7)$ & $\begin{array}{l}\text { Coadjuvante no tratamento de } \\
\text { hiperlipidemia, hipertensão arterial } \\
\text { leve, dos sintomas de gripes e } \\
\text { resfriados e auxiliar na prevenção da } \\
\text { aterosclerose }{ }^{4,9} \text {. }\end{array}$ \\
\hline $\begin{array}{c}\text { Maracujá } \\
\text { Passiflora alata Curtis. } \\
\text { Passiflora incarnata } L .\end{array}$ & 01 (P4) & Ansiolítico e sedativo leve 4,9 . \\
\hline $\begin{array}{c}\text { Cidreira } \\
\text { Cymbopogoncitratus } \\
\text { (DC.) Stapf }\end{array}$ & 01 (P5) & $\begin{array}{l}\text { Antiespasmódico, ansiolítico e } \\
\text { sedativo leve }{ }^{4} \text {. }\end{array}$ \\
\hline
\end{tabular}

Fonte: Brasil ${ }^{4,9}$.

A descrição da utilização das plantas medicinais para doenças respiratórias utilizada pelos pacientes segue conforme os relatos: 
P1- "O mel, folha de laranja, guaco, hortelã".

P2- "Uso a folha de laranja".

P3 - "Tomo hortelã e guaco".

P4- "Tomo camomila, maracujá".

P5- "Cidreira e guaco".

P6- "Tomo o guaco, hortelã, camomila".

P7- "Tomo o alho".

P8- "Tomo um chá do mel de abelha".

P9- "Eu faço um chá de camomila".

P10- "Só um chá de camomila à noite, para dormir melhor".

Destaca-se que o guaco é considerado expectorante e a tintura do alho é considerada coadjuvante no tratamento dos sintomas da gripe e resfriados; camomila, laranja, maracujá e cidreira são ansiolíticos e sedativos leves; a hortelã é antiespasmódica ${ }^{4}$.

\section{Indicação do uso da planta medicinal}

A maioria dos pacientes relata que o conhecimento sobre as plantas medicinais tem origem no conhecimento popular e advém primordialmente da família:

P1- "O conhecimento vem da família, minha mãe me passou o que era bom".

P2- "Na minha família usavam, uma prima minha disse que era bom".

P5- "Minha família usa, minha avó falava que era bom tomar chá".

P6- "Minha esposa participa da Pastoral da Saúde, daí ela sabe bem".

P7- "Sei por conhecimento popular, minha família".

P10- "Por conhecimento da família mesmo".

A utilização de plantas medicinais é tão antiga quanto a própria espécie humana, acompanhando o ser humano no seu processo de evolução, sendo 
Artigo Original

\section{Atenção à Saúde}

fundamentada no acúmulo de informações repassadas de geração a geração e utilizada como forma de tratamento e cura ${ }^{2,6,8,24-27}$.

Durante muitos anos, os remédios naturais e, sobretudo, as plantas medicinais foram a principal alternativa para a cura de diversas doenças e para a fabricação de remédios farmacêuticos ${ }^{24}$. A forma de tratamento natural é extremamente eficaz para algumas patologias, mas vem perdendo espaço para uma medicina sintética, devido ao dia-a-dia corrido, em um cotidiano acelerado, na qual a praticidade é compensatória ${ }^{24}$.

A utilização de plantas medicinais vem se descaracterizando devido à grande quantidade de medicamentos sintéticos introduzidos no mercado e à falha na passagem de informações para as próximas gerações, possivelmente devido à falta de interesse das novas gerações em relação ao conhecimento tradicional e migração das pessoas para grandes centros urbanos, onde a disponibilidade desse tipo de matéria-prima é menor ${ }^{24}$.

Destaca-se na fala do paciente P6 que a esposa participa da Pastoral da Saúde, o que possibilita um maior conhecimento sobre as plantas medicinais para 0 uso familiar.

Os pacientes P8 e P9 relataram que utilizam a planta medicinal por preferência e indicação própria:

P8-"Tomo chá de mel porque eu gosto, ninguém falou que era bom".

P9- "Eu tomo por minha conta mesmo, ninguém indicou".

Os pacientes P3 e P4 tiveram indicação do uso pelo médico:

P3 - "O médico mandou eu tomar, falou que era bom".

P4- "O médico que me trata falou que é bom".

Por causarem uma imensa gama de limitações aos pacientes, as doenças respiratórias crônicas devem ser assistidas de perto por uma equipe multidisciplinar, de modo interdisciplinar. As limitações físicas, intelectuais e emocionais que surgem com a doença podem interferir tanto na vida do doente quando na dos seus familiares. Os cuidados ao paciente e sua família devem ser prestados por uma 
Artigo Original

Atenção à Saúde

equipe composta de médicos, enfermeiros, fisioterapeutas, psicólogos, nutricionistas, terapeutas ocupacionais, entre outros, a fim de alcançar o nível máximo de autonomia e funcionalidade dos usuários. Essa equipe deve ser bem competente, articulada e corresponsabilizar suas ações, de modo que um profissional dê continuidade ao trabalho do outro, ambos com o intuito de melhorar a qualidade de vida do usuário. Buscando, dessa maneira, aprimorar o atendimento proporcionado ao paciente para que o mesmo possa estar convivendo e enfrentando os desafios decorrente do processo saúde-doença ${ }^{28}$.

A formação e a qualificação dos profissionais de saúde são desafios enfrentados para a inserção e utilização de plantas medicinais no âmbito da saúde. O Programa de Plantas Medicinais e Fitoterapia coloca em ênfase a necessidade do empoderamento da população e dos profissionais da saúde em relação à utilização de plantas medicinais, fornecendo assim orientações seguras e estimulando o autocuidado e autonomia dos indivíduos ${ }^{3}$.

Conforme a Política para Plantas Medicinais e Fitoterapia, destaca que se faz necessário a formação e educação permanente dos profissionais de saúde em plantas medicinais e fitoterapia, o aumento e fortalecimento da participação da população, com o intuito de disseminar os saberes, aumento das pesquisas e trabalhos científicos valorizando a biodiversidade do país e promoção do uso racional das matérias-primas para produção e consumo de plantas medicinais e fitoterápicos ${ }^{2,3,5,6,25}$.

É indispensável que os profissionais de saúde, além de possuírem o conhecimento científico sobre a utilização de plantas medicinais, conheçam o espaço, a estrutura, os hábitos de vida da população em que irão prestar os cuidados, procurando compreender a maneira de pensar e agir dos indivíduos frente aos seus problemas de saúde, tornando mais fácil, dessa maneira, a comunicação, possibilitando um cuidado mais resolutivo e rápido voltado para as necessidades destas populações ${ }^{8}$.

\section{Forma de utilização das plantas medicinais para doenças respiratórias}

Todos os pacientes relataram preparar as plantas medicinais para doenças respiratórias em forma de chá: 
P1- "Uso na forma de chá".

P3 - "Minha filha faz um chá para mim tomar de noite".

P4- "Eu faço um chá e bato alguns sem açúcar".

P6- "Faço um chá com as folhas".

P7- "Em forma de chá".

P9- "Tomo como chá mesmo".

A forma de preparação das plantas medicinais, segundo a literatura, está indicada no Quadro 3.

Quadro 3. Forma de preparação das plantas medicinais, segundo Formulário de Farmacopéia Brasileira.

\begin{tabular}{|c|c|}
\hline Guaco & $\begin{array}{l}\text { Parte utilizada: Folhas secas. Modo de usar: Infusão: } 3 \mathrm{~g} \text { em } \\
150 \mathrm{~mL} \text { água. Tomar } 150 \mathrm{~mL} \text { do infuso, logo após o preparo, duas } \\
\text { vezes ao dia }{ }^{4} \text {. }\end{array}$ \\
\hline Hortelã & $\begin{array}{l}\text { Parte utilizada: Folhas e sumidades floridas. Modo de usar: } \\
\text { Infusão: } 1,5 \mathrm{~g} \text { em } 150 \mathrm{~mL} \text { água. Tomar } 150 \mathrm{~mL} \text { do infuso, } 10 \\
\text { minutos após o preparo, duas a quatro vezes ao dia }{ }^{4} \text {. }\end{array}$ \\
\hline Laranja & $\begin{array}{l}\text { Parte utilizada: Flores secas. Modo de usar: Infusão: } 1-2 \mathrm{~g} \text { em } \\
150 \mathrm{~mL} \text { água. Tomar } 150 \text { a } 300 \mathrm{~mL} \text { do infuso após } 5 \text { minutos do } \\
\text { preparo, de preferência no início da noite }{ }^{4} \text {. }\end{array}$ \\
\hline Alho & $\begin{array}{l}\text { Parte utilizada: Bulbilhos secos. Modo de usar: Tintura: } 20 \mathrm{~g} \text { em } \\
100 \mathrm{ml} \text { de álcool } 45 \% \text { p/p q.s.p. Tomar } 50 \text { a } 100 \text { gotas }(2,5 \text { a } 5 \mathrm{~mL}) \\
\text { da tintura diluídas em } 75 \mathrm{~mL} \text { de água, duas a três vezes ao dia }{ }^{4} \text {. }\end{array}$ \\
\hline Maracujá & $\begin{array}{l}\text { Parte utilizada: folhas secas. Modo de usar: Infusão: } 3 \mathrm{~g} \text { em } \\
\text { 150mL de água. Tomar } 150 \mathrm{~mL} \text { do infuso, } 10 \text { a } 15 \text { minutos após o } \\
\text { preparo, duas a quatro vezes ao dia }{ }^{4} \text {. }\end{array}$ \\
\hline Cidreira & $\begin{array}{l}\text { Parte utilizada: Folhas secas. Modo de usar: Infusão: } 1-3 \mathrm{~g} \text { em } \\
150 \mathrm{~mL} \text { de água. Tomar } 150 \mathrm{~mL} \text { do infuso, } 5 \text { minutos após o } \\
\text { preparo, duas a três vezes ao dia }{ }^{4} \text {. }\end{array}$ \\
\hline
\end{tabular}

Fonte: Brasil ${ }^{4}$. 
Conforme o Formulário de Farmacopéia Brasileira ${ }^{4}$, a decocção é gerada a partir da ebulição da droga vegetal em água potável por um tempo determinado. É normalmente indicada para ser utilizada em drogas vegetais com consistência rígida, como cascas, raízes e sementes. A infusão consiste em colocar água fervente sobre a droga vegetal, e em seguida tampar o recipiente por tempo determinado. Método normalmente utilizado com partes de drogas vegetais menos rígidas, como é o caso de folhas, frutos e flores. Já a maceração consiste em um processo de manter a droga pulverizada conforme as indicações da fórmula, em contato com o líquido extrator, devendo-se manter a agitação diária por no mínimo sete dias seguidos. 0 preparo deve ser realizado em um recipiente que não permita o contato com a luz, em temperatura ambiente. Após a maceração, se distribui a mistura em um litro e lavam-se os resíduos restantes no filtro com líquido extrator, de forma a obter o volume inicial indicado na fórmula 4 .

A partir da entrevista com os pacientes observou-se a dificuldade dos mesmos para relatar com clareza como preparam os chás, se por infusão ou decocção. Considera-se que a equipe multiprofissional deve ter conhecimento sobre as plantas medicinais utilizadas pelos pacientes no grupo, para orientar de forma adequada sobre as indicações, contraindicações, modo de preparo e interação medicamentosa das plantas medicinais.

\section{Armazenamento das plantas medicinais}

Os pacientes relataram que armazenam as plantas medicinais no próprio sachê:

P1- "Deixo em casa dentro de um saquinho".

P2- "Só nos pacotinhos do mercado".

P3 - "Deixo dentro do saquinho que ele vem".

P9- "Deixo dentro do saquinho do chá mesmo".

P10- "Só nos pacotinhos do mercado".

Os pacientes P4, P5 e P6 adquirem as plantas medicinais no quintal de casa; sendo que os pacientes P5 e P6 armazenam as plantas em pote: 
P4- "Eu pego no quintal quando precisa".

P5- "Pego quando preciso no quintal ou seco na sombra e guardo nos potes em casa".

P6- "Deixo secar e guardo as folhinhas em um pote em casa, quanto não tem na horta".

Somente os pacientes P7 e P8 armazenam as plantas medicinais na geladeira, além de guardarem no armário ou fruteira:

P7- "Deixo na geladeira, ou na fruteira de casa".

P8- "Eu guardo na geladeira ou no armário lá de casa".

O armazenamento de plantas medicinais deve ser feito respeitando algumas regras básicas, para que as plantas não estraguem ou percam suas propriedades naturais. As que são plantadas no quintal devem ser colhidas no período da manhã, e serem bem higienizadas, as plantas secas devem ser armazenadas em recipientes que facilitem a visualização, limpos e secos. Todas as plantas devem estar bem protegidas e longe de qualquer resíduo que possa contaminar as ervas, atentandose sempre a sua validade e sinais como mofo e odor fétido que podem impossibilitar o uso 29 .

As plantas medicinais devem ser armazenadas depois de colhidas em locais frescos e que não apresentem umidade, protegidas da ação dos raios solares, água e insetos, para manter seu estado de conservação adequado para o uso. Desta maneira conservando e mantendo o seu princípio ativo, que é o responsável pelo efeito benéfico causado no organismo e as deixando livres de contaminação ${ }^{30}$.

Algumas plantas medicinais precisam de cuidados específicos para manter a eficácia. O plantio no solo e época do ano adequado, com quantidade de água, luz, calor, umidade e quantidade de ventos diretos e excessivos, são fatores que influenciam diretamente a planta ${ }^{31}$.

\section{Local de aquisição das plantas medicinais}


As plantas medicinais são adquiridas principalmente na farmácia ou no mercado (P1; P2; P3; P7; P8; P9; P10); no quintal de casa (P1; P4; P5; P6; P7); com os vizinhos e amigos (P2; P3; P4; P5; P6), conforme destacado nos relatos:

P1- "Se não tem no quintal de casa, vou na farmácia de manipulação comprar que lá tem".

P2- "Pego as folhas dos meus amigos, ou vou lá e compro na farmácia".

P3 -"Minha filha compra no mercado ou pede para algum vizinho que tenha em casa".

P5- "Pego nos fundos da minha casa, ou vou pegar nos vizinhos".

P6- "Pego na horta, com os vizinhos. Não preciso comprar nada".

P9- "Compro no mercado".

Para o uso racional das plantas medicinais, é necessário que os usuários estejam orientados sobre o plantio de mudas ou como adquirir em uma farmácia ou mercado, e sua forma de utilização correta. Plantas possuem nomes populares que costumam variar de região para região, um cuidado muito importante é ter conhecimento sobre o nome científico da planta, evitando assim engano e uso inapropriado. Informações básicas sobre indicação de uso, local da coleta, conhecer a planta a ser usada, melhor horário para coleta, forma de preparo, quantidade a ser utilizada são indispensáveis para a utilização de forma benéfica ${ }^{32}$.

O local de aquisição das plantas é de extrema importância, sendo ele um mercado ou até o quintal de cada. As plantas precisam estar em um local limpo e livre de contaminações, pois as mesmas estão sujeitas a interagir com o meio em que vivem, dessa forma perdendo suas propriedades medicinais e até tornando-se prejudiciais à saúde.

\section{As orientações recebidas pelos pacientes sobre as plantas medicinais da equipe multiprofissional da saúde}

Alguns dos pacientes não receberam orientação da equipe multiprofissional sobre a utilização das plantas medicinais, segundo os relatos: 
P1- "Nunca recebi orientação sobre plantas".

P2- "Nunca recebi orientações".

P8- "Nunca me falaram nada sobre isso".

P9- "Nunca recebi orientação sobre plantas".

P10- "Nunca recebi orientações".

Os pacientes P5 e P6 tiveram acesso às informações sobre o uso das plantas através da Pastoral da Saúde:

P5- "Fiz o curso da Pastoral da Saúde quando era mais nova há muitos anos, quem passou foi a professora do curso da Pastoral naquele tempo".

P6- "Não sei, mas minha esposa fez o curso da Pastoral, ela recebeu palestras com as irmãs do hospital [...]".

Os pacientes $\mathrm{P} 3, \mathrm{P} 4$ e $\mathrm{P} 7$ receberam informações sobre as plantas através do médico. O paciente P3 acessou informações a partir da Internet:

P3 - "O médico falou que era bom tomar, e minha filha olhou na Internet também".

P4- "O médico falou que faz bem tomar".

P7- "Uma médica falou que era bom tomar chá assim".

Constata-se no relato do paciente $\mathrm{P} 3$ que a Internet facilita o acesso às informações sobre cuidados com a saúde, o que possibilita um maior conhecimento dos pacientes e familiares sobre assuntos relacionados à utilização de plantas medicinais.

Conforme o Programa Nacional de Plantas Medicinais e Fitoterápicos, as leis que regulamentam a utilização de plantas medicinais devem salvar, preservar, apoiar, guardar os conhecimentos, práticas e saberes tradicionais e populares em plantas medicinais e produtos para saúde que possuem sabedoria ancestral, conhecimento popular que foi passado de geração após geração. As características que comprovam a eficácia desses produtos oriundos dos antigos conhecimentos são referendadas pela tradição. Assim, com incentivo, apoio e fomento apropriado, 
aproximam-se os conhecedores desses saberes com a realidade vivenciada hoje no Sistema Único de Saúde ${ }^{6,25}$.

Para incentivar a formação e a capacitação de recursos humanos para o desenvolvimento de pesquisas, tecnologias e inovação em plantas medicinais e fitoterápicos são necessárias ações que visem a qualificação dos profissionais de saúde e demais envolvidos na produção e uso de plantas medicinais e fitoterápicos. Essas ações podem ser realizadas por meio de sugestão do MEC sobre inclusão de disciplinas específicas na formação de profissionais de saúde e outros envolvidos na produção e uso de plantas medicinais e fitoterápicos, programas que visam promover a capacitação técnica e educação permanente em plantas medicinais e fitoterápicos, dos profissionais de saúde do SUS, em conformidade com a Política Nacional de Educação Permanente. A ampliação das opções terapêuticas ofertadas aos usuários do SUS, garantindo acesso à plantas medicinais e fitoterápicos, com segurança, eficácia e qualidade, nos diferentes níveis de complexidade do Sistema, com ênfase na atenção básica, por meio de ações de prevenção de doenças e de promoção e recuperação da saúde, é uma importante estratégia, visando à melhoria da atenção à saúde da população e à inclusão social ${ }^{6,25}$.

O Brasil é o país que apresenta a maior biodiversidade mundial, as plantas são parte integrante e fundamental dessa diversidade e são a matéria-prima para a fabricação de medicamentos em geral e fitoterápicos. Elas também são utilizadas por meio de práticas passadas de geração após geração, como remédios caseiros e comunitários, práticas essas que fazem do Brasil um país com rica diversidade cultural e étnica, que deve ser preservada e respeitada através de políticas. Promovendo, dessa maneira, o acesso seguro e o uso racional das plantas medicinais e fitoterápicos, promovendo o uso sustentável e o desenvolvimento da cadeia produtiva e da indústria nacional ${ }^{2}$.

O enfermeiro tem papel fundamental na utilização de plantas medicinais, e no processo saúde-doença da comunidade em que o mesmo atua como coordenador de equipe, devendo exercer ações de prevenção e promoção à saúde, construindo vínculos com a comunidade, a fim de melhorar a assistência prestada. Esse contato possibilita ao profissional conhecer a cultura e o saber popular de determinada comunidade. $O$ conhecimento restrito e informal que os profissionais enfermeiros têm, sobre a utilização de fitoterápicos na atenção básica, faz com que 
os mesmos encontrem grandes dificuldades para a implantação de práticas fitoterápicas, e desafios como falta de valorização por parte da gestão e da própria equipe de saúde. É de suma importância que os profissionais enfermeiros saibam orientar e assistir a população quanto à utilização de fitoterápicos, porém esse conhecimento se faz deficiente nos dias atuais. Os autores apontam a falta de conhecimento científico, por deficiência no sistema acadêmico ou por falta de interesse na área como os motivos para tal fato ${ }^{33}$.

\section{Orientação profissional sobre interação medicamentosa com as plantas medicinais utilizadas}

Os pacientes $\mathrm{P} 1, \mathrm{P} 2, \mathrm{P} 3, \mathrm{P} 4, \mathrm{P} 7, \mathrm{P} 8$ e $\mathrm{P} 9$ relataram não ter recebido orientação profissional sobre interação medicamentosa com as plantas medicinais utilizadas. Os pacientes $\mathrm{P} 5, \mathrm{P} 6$ e $\mathrm{P} 10$ não souberam relatar.

Os fitoterápicos e plantas medicinais vêm sendo incluídos nos programas de farmacovigilância e reconhecidos nos últimos anos por vários países da Europa, onde diversas plantas foram submetidas a testes da farmacovigilância e muitas delas foram retiradas do mercado por apresentarem efeitos tóxicos e riscos para os seres humanos. Com o crescente aumento no número de reações adversas reportadas, é possível que a causa seja justificada pelo aumento do interesse da população pelas terapias naturais, sem o devido conhecimento. Se faz necessário a divulgação de um programa de farmacovigilância de fitoterápicos entre os profissionais de saúde, principalmente para profissionais que atendam na atenção primária à saúde, assim evitando erros como toxidade e contra-indicação ou uso concomitante de outros medicamentos ${ }^{34}$.

\section{CONSIDERAÇÕES FINAIS}

As principais plantas utilizadas pelos pacientes que participam do Grupo de Reabilitação Pulmonar foram camomila, boldo, guaco, cidreira, hortelã, folha de laranja e mel.

Dos pacientes que utilizam plantas medicinais para doenças respiratórias, quatro utilizam guaco, que é considerado expectorante e broncodilatador, e um 
paciente utiliza o alho, que é considerado coadjuvante no tratamento dos sintomas da gripe e resfriados, conforme a Farmacopéia Brasileira. No modo de preparo, o alho é utilizado em forma de chá, conforme relatado pelo paciente P7, e não de tintura, conforme indicação da Farmacopéia Brasileira.

Quatro pacientes, ainda, utilizam a camomila e um paciente a cidreira, que são antiespasmódicos e sedativos; dois pacientes utilizam a laranja e um o maracujá, que são ansiolíticos e sedativos; três pacientes utilizam a hortelã, que possui ação antiespasmódica, segundo a Farmacopéia Brasileira.

A forma utilizada das plantas medicinais pelos pacientes geralmente está de acordo com as indicações da ANVISA. Os pacientes demonstraram dificuldade para relatar como ocorre a preparação das plantas medicinais.

Considera-se primordial a revalorização do uso de plantas medicinais pela equipe multiprofissional de saúde, em concordância com as diretrizes da Política Nacional de Práticas Integrativas e Complementares no SUS (PNPIC) e Programa Nacional de Plantas Medicinais e Fitoterápicos. Para tanto, faz-se necessário incluir nos cursos de graduação à saúde a inserção de disciplinas que contemplem conteúdos voltados à plantas medicinais e fitoterápicos.

Sugere-se novos estudos direcionados aos profissionais da saúde que atuam na atenção básica e hospitalar, principalmente voltado a atuação do enfermeiro no cuidado e orientação aos usuários da rede que utilizam plantas medicinais.

\section{REFERÊNCIAS}

1 Brasil. Ministério da Saúde. Secretaria de Atenção à Saúde. Departamento de Atenção Básica. Doenças respiratórias crônicas. Brasília: Ministério da Saúde, $2010.160 \mathrm{p}$.

2 Brasil. Ministério da Saúde. Secretaria de Atenção à Saúde. Departamento de Atenção Básica. Portaria 971, de 03 de maio de 2006. Aprova a Política Nacional de Práticas Integrativas e Complementares no SUS. Brasília: Ministério da Saúde, 2006.

3 Brasil. Ministério da Saúde. Secretaria de Atenção à Saúde. Departamento de Atenção Básica. Práticas integrativas e complementares: plantas medicinais e fitoterapia na Atenção Básica. Brasília: Ministério da Saúde, 2012. 156 p. 


\section{Atenção à Saúde}

$4 \quad$ Brasil. Agência Nacional de Vigilância Sanitária. Formulário de Fitoterápicos da Farmacopéia Brasileira. Brasília: Anvisa, 2011. 126p.

5 Brasil. Ministério da Saúde. Secretaria de Atenção à Saúde. Departamento de Atenção Básica. Política nacional de práticas integrativas e complementares no SUS: atitude de ampliação de acesso. 2. ed. Brasília: Ministério da Saúde, 2013. 96 p.

6 Brasil. Ministério da Saúde. Secretaria de Ciência, Tecnologia e Insumos Estratégicos. Departamento de Assistência Farmacêutica. Política e Programa Nacional de Plantas Medicinais e Fitoterápicos. Brasília: Ministério da Saúde, 2016. $190 \mathrm{p}$.

7 Lopes GAD, Feliciano LM, Diniz RES, Alves MJQF. Plantas medicinais: indicação popular de uso no tratamento de Hipertensão Arterial Sistêmica (HAS). Rev. Ciência em Extensão, São Paulo, 2010 jan; 6(2):143-155.

8 Piriz MA, Mesquita MK, Cavada CT, Palma JS, Ceolin T, Heck RM. Uso de plantas medicinais: impactos e perspectivas no cuidado de enfermagem em uma comunidade rural. Rev. Eletr. Enf., Pelotas. 2013 out/dez;15(4):1000-6.

9 Brasil. Ministério da Saúde. Agência Nacional de Vigilância Sanitária instrução normativa $\mathrm{n}^{\circ} 02$ de 13 de maio de 2014. [Acesso em: 2017 mar. 27]. Disponível em: <http://bvsms.saude.gov.br/bvs/saudelegis/anvisa/2014/int0002_13_05_2014.pdf>.

10 Brasil. Conselho Nacional de Saúde. Resolução no 466, de 12 de dezembro de 2012. [Acesso em: 2015 nov.5]. Disponível em:

<http://conselho.saude.gov.br/resolucoes/2012/Reso466.pdf>.

11 Minayo MC S. Pesquisa social: teoria, método e criatividade. 28 ed. Petrópolis, RJ: Vozes, 2009. 108 p.

12 Brasil. Ministério da Saúde. Secretaria de Atenção à Saúde. Departamento de Atenção Básica. Diretrizes para o cuidado das pessoas com doenças crônicas nas redes de atenção à saúde e nas linhas de cuidado prioritárias. Brasília: Ministério da Saúde, 2013. 28 p.

13 Brasil. Ministério da Saúde. Secretaria de Atenção à Saúde. Departamento de Atenção Básica. Estratégias para o cuidado da pessoa com doença crônica: diabetes mellitus. Brasília: Ministério da Saúde, 2013. 160 p.

14 Baldi BG, Pereira CAC. Diretrizes de Doenças Pulmonares Intersticiais da Sociedade Brasileira de Pneumologia e Tisiologia. J Bras Pneumol., 2012; v.38(supl.2): S1-S133.

15 Camelo-Nunes IC, Solé D. Rinite alérgica: indicadores de qualidade de vida. J. Bras. Pneumol. 2010 fev; 36(1): 124-133. 
16 Lacerda MA, Teixeira MG; Pimenta Júnior F. Protocolo da Rinite Alérgica. Belo Horizonte: Prefeitura de Belo Horizonte, 2012. 25 p.

17 Marchiori RC, Susin CF, Dal Lago L, Felice CD, Silva DB, Severo MD. Diagnóstico e tratamento da DPOC exacerbada na emergência. Rev. AMRIGS, Porto Alegre, 2010 abr/jun.; 54 (2): 214-223.

18 Bassi D. Doença Pulmonar Obstrutiva Crônica: considerações sobre deglutição e qualidade de vida. 2014. 86 f. Dissertação (Mestrado Profissional) Universidade Federal de Santa Catarina, Centro de Ciências da Saúde. Programa de Pós Graduação Multidisciplinar em Saúde. Florianópolis, SC, 2014.

19 Losso LC. Enfisema Pulmonar[Internet]. CREMESP, 2012. [Acesso em: 2015 nov.5]. Disponível em: < http://www.luiscarloslosso.com.br/pdf/EnfisemaPulmonar.pdf>.

20 Brandão DS. Tratamento do enfisema pulmonar avançado: cirurgia redutora do volume pulmonar ou broncoscopia. Pulmão Hospital Regional da Asa Norte, Rio de Janeiro, 2014 jan.; 23(1): 31-35.

21 Ribeiro FSN (coord.) O mapa da exposição à sílica no Brasil. Rio de Janeiro: UERJ, Ministério da Saúde, 2010. 94 p.

22 Andrade JP, Nobre F. VI Diretrizes Brasileiras de Hipertensão. Arq Bras Cardiol, 2010; 95(supl.1):1-51, 2010.

23 Valle LS, Souza VF, Ribeiro AM. Estresse e ansiedade em pacientes renais crônicos submetidos à hemodiálise. Estudos de Psicologia, Campinas, 2013mar.; 30(1):131-138. DOI https://dx.doi.org/10.1590/S0103-166X2013000100014

24 Quevedo MD, Gonçalves RF, Gonzales F. O conhecimento sobre a utilização de plantas medicinais em dois municípios do litoral de São Paulo, SP. Rev. Ceciliana, São Paulo, 2011 dez; 2(3):35-39.

25 Brasil. Ministério da Saúde. Secretaria de Ciência, Tecnologia e Insumos Estratégicos. Departamento de Assistência Farmacêutica e Insumos Estratégicos. Programa Nacional de Plantas Medicinais e Fitoterápicos. Brasília: Ministério da Saúde, 2009.136 p.

26 Pereira SM, Almeida TWF, Dias ASF, Pinheiro CT, Sousa VR, Silva GRC et al. Levantamento do potencial medicinal das plantas produzidas e dispensadas na Pastoral da Saúde de Itapuranga/GO. Rev. Faculdade Montes Belos (FMB) 2015; 8(1): 22-26.

27 Silva MR. A utilização do conhecimento de plantas medicinais como ferramenta para estimular a preservação ambiental. Monografias Ambientais. REMOA/ UFSM, mar.2012; 6(6):1354-1380. 


\section{Artigo Original}

\section{Atenção à Saúde}

28 Dias TM, Pinno C, Schmitt FV, Rosat LI, Gonçalves MP. Cuidado multiprofissional ao pneumopata crônico: promovendo a integralidade do sujeitousuário[Internet], 2016. [Acesso em: 2016 maio 26]. Disponível em: <http://www.unifra.br/eventos/forumfisio/Trabalhos/5136.pdf>.

29 Badke MR, Budó MLD, Silva FM, Ressel LB. Plantas medicinais: o saber sustentado na prática do cotidiano popular. Esc. Anna Nery. 2011 mar.; 15(1): 132139. DOI http://dx.doi.org/10.1590/S1414-81452011000100019.

30 Nascimento W MC, Melo OF, Silva IF, Souza FL. Plantas medicinais e sua utilização pelas comunidades do município de Sobral, Ceara. Sanare, Sobral Ce, 2013 jun.; 12(01):46-53.

31 Rossato AE, Pierini MM, Amaral PA, Santos RR, Zanette VC (Org.). Fitoterapia racional: aspectos taxonômicos, agroecológicos, etnobotânicos e terapêuticos. Florianópolis: DIOESC, 2012. v. 1.

32 Pereira MJL, Thé PMP, Leal LKAM. Promoção do uso racional de plantas medicinais em creche-escola no Ceara[Internet],2016. [Acesso em: 2016 jan. 01]. Disponível em: <http://www.convibra.org/upload/paper/2014/55/2014_55_9504.pdf>.

33 Sampaio LA, Oliveira DR, Kerntopf MR, Brito Júnior FE, Menezes IRA. Percepção dos enfermeiros da estratégia saúde da família sobre o uso da fitoterapia. Rev Min Enferm. 2013 jan/mar; 17(1): 76-84. DOI:10.5935/14152762.20130007.

34 Silveira PF, Bandeira MAM, Arrais PSD. Farmacovigilância e reações adversas às plantas medicinais e fitoterápicos: uma realidade. Rev. bras. farmacogn. $2008 \mathrm{dez} ; \quad 18(4)$ : 618-626. http://dx.doi.org/10.1590/S0102$695 \times 2008000400021$. 